\title{
Challenges in Applying Objects to Large Systems
}

\author{
Juha-Markus Aalto \\ Nokia Telecommunications, Cellular Systems \\ Mobile Switching, Network Management Systems \\ Hatanpäänvaltatie 36 A, 33100 Tampere, Finland \\ Juha-Markus.Aalto@ntc.nokia.com
}

\begin{abstract}
Object-orientation seems to be the winning approach in the war of software development paradigms. Compilers for the $\mathrm{C}$ programming language have been largely updated to support the object-oriented $\mathrm{C}++_{+}$, modern CASE tools and software development methods are designed for object-oriented approaches, and more and more software development organizations believe that objects help them to cut costs and improve software quality.
\end{abstract}

A natural consequence of the popularity of object-oriented methods and tools is that today they are also used for developing large systems. This paper discusses how object technology affects the success factors of an organization developing large systems. This paper is based on experiences in institutionalizing objectoriented technology in the development organization of a large network management system called the Nokia OMC.

\section{$1 \quad$ Introduction}

Typically, examples in software engineering books show how a software engineering methodology can be applied to a relatively small application. This is usually done in order to keep the examples readable. Examples serve to clarify the author's ideas of how a specific approach can and should be applied under certain circumstances. The suggested text book approaches usually provide more or less half-baked ideas rather than real solutions to software process challenges. Unfortunately, many organizations develop also large systems on the basis of these books although a number of issues need to be solved before any of the proposed methods can successfully be applied to large projects.

The development of large systems differs considerably from that of small ones. The main differences are:

- Large systems are complex and require so much effort that they cannot be created by one individual; organized teams are needed. Good communication is essential both between and within these teams.

- Large systems are typically developed incrementally: New features are developed by reusing existing software. Enhancements to existing features are common; 
changes in customers' business needs often bring about new software requirements.

- Large systems are often mission critical systems, such as air traffic control systems and telecommunications infrastructures. They are extremely important for the customer's business, and the quality of the delivered systems needs to be particularly high to ensure maximum reliability.

- The customers of companies developing large systems are typically other companies, not consumers. Therefore, the large systems need to fit into the business activity of the user organization.

The Nokia OMC is one of the leading network management systems for managing digital GSM networks. It is an example of a large system developed using object technology. It consists of more than one hundred concurrently running processes with over 1.000.000 LOC written in C++. The development of the system takes place in parallel main projects that each have several sub-projects. Each main project produces a release which consists of features that implement an increment to the functionality of the existing system. The size of an increment is typically $200.000-300.000 \mathrm{LOC}$. The Nokia OMC is a mission critical system for network operators who use it in building and monitoring their networks. Therefore, the requirements for software quality are high, and the functionality of the system needs to closely match the business activity of the operators.

A talented individual or two can implement outstanding applications using any methods (or none) if the problem domain is well understood and the application size is in the range of $10.000-100.000$ LOC. Large systems, such as the Nokia OMC require a wide range of knowledge about the problem domain, and the technical complexity increases so much that the heroic efforts of one or a few gurus are not enough. The success of an organization developing large systems seems to mostly depend on the following factors:

- a competitive product matching the customers' needs,

- an effective and efficient process,

- feasible technology used in product and in its development,

- capable and motivated personnel, and

- carefully maintained software assets.

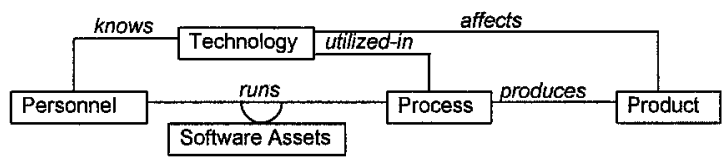

Fig. 1. Important success factors of a system supplier.

The relationships of the success factors are depicted in Figure 1. Perhaps the most important factor is that the product, in other words, the system to be developed, matches the customer's needs. A prerequisite for this is that the process that 
implements the system is effective in ensuring that all requirements are caught and implemented efficiently. Furthermore, the efficient implementation of the process is highly dependent on the skills and motivation of the personnel. Another basic competitive requirement is that the right technology is used in the product and in its development. The software assets of the system, such as code and documentation, are the results of the process that will be reused several times in the future. That property needs to be kept in good shape so that it can be further processed and sold to customers over several years.

This paper views the applicability of object-oriented approaches to software engineering from the viewpoint of the above-mentioned success factors that are important in developing large systems. Some problems recognized in popular methodologies, such as Object Modeling Technique (OMT) [13], Fusion [4] and Object-oriented Software Engineering [10], are highlighted. Solutions for these problems have been developed by Nokia Telecommunications and Nokia Research Center. The resulting methodology is called OMT++ [2], and it is based on the above mentioned methodologies.

\section{Product Viewpoint}

Nobody wants to buy a product that does not fulfill the needs of its users. A company developing such a product is soon out of the market place. The most critical phase in software development is therefore the requirements definition phase, during which the requirements for the system to be developed are identified, analyzed and documented. If the system is purchased by another company, which is typical for large systems, the system should make a part of the customer's business activity automatic or more manageable in order to improve the performance of the company. To be able to analyze and evaluate customer requirements, we need to understand the business activity of the customer.

Business process re-engineering (BPR) is an approach to business development in which all business related activities of a company are re-thought. Typically, the operations of the company are modeled as chains of activities that add value to the customer's business. These chains are called core business processes, and the idea is to streamline these processes and automate them using information technology. Typical goals are to dramatically shorten the production lead times or to reduce costs.

Jacobson has described how a company can re-engineer its operations using a modified use case approach of the Object-oriented Software Engineering (OOSE) method [10] called Object-oriented Business Engineering [11]. Business modeling techniques used in business engineering can also be utilized in software development, not only in business process re-engineering. A software supplier can 'reverse engineer' the relevant business processes of the customers in order to understand their business environment. The results can be utilized in software requirements analysis so that the software features can be well integrated in customers' business needs. Our on-going 
work has already verified the feasibility of a similar approach: It is possible to establish a continuous, traceable path from the customers' business processes to user interface actions, as depicted in Figure 2.

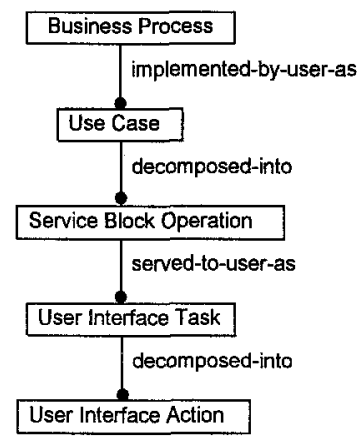

Fig. 2. From business processes to user interface actions with OMT++. The service blocks are sub-systems that are responsible for a set of closely related services.

It is likely that new object-oriented approaches to business area analysis will be published in the near future in addition to Object-oriented Business Engineering, since the object-oriented methods offer suitable modeling facilities for this purpose. It is also likely that business area analysis will constitute a front end for the requirements definition of other methods, too, in addition to OOSE and OMT++.

Business process modeling helps mostly in finding process related functional requirements. However, they are not the only requirements for software: there are also non-functional ones which correspond the requirements for the technical characteristics of the system, such as capacity, response times, reliability, and maintainability. The non-functional requirements are mostly related to the technical constraints of the target environment. However, quite a few of them can be derived from attributes of business processes such as business volumes, frequencies of operations, and the number of persons related to each activity.

Most of the object-oriented methods do not address non-functional requirements in particular, although they constitute an essential part of the specification work of applications. The hierarchy of customer needs (analogous to Maslow's one for human beings) for a new software system seems to be as follows:

1. First, the most essential functionality must exist and provide some useful results.

2. When the system is able to provide some results, the requirements concentrate on the stability of the system for long periods of time.

3. The capacity of the reliable and stable system must then be adequate and scaled up as the use and number of users increase. 
4. In order to make full use of the capacity, the performance of the system must meet the users' daily needs.

5. When the system is reliable and fast enough to deal with its capacity, the user interface must be sufficiently flexible to ensure effective and pleasant use.

For instance, the life-cycle of many CASE tools follows the pattern above. Of course, the above list is a simplified view of customer needs, but it shows that there are several things to be analyzed and designed in addition to the objects and functions of the system. It is important to notice that software requirements are only part of all requirements known as the system requirements, which may be related to hardware, for instance. Another way to classify requirements is to divide them into customer/user-oriented software requirements (C-requirements) and developeroriented requirements (D-requirements) [3]. The C-requirements provide the customer with a description of all the important requirements that are adequate for commitment to software development. The D-requirements are typically elaborated from C-requirements, and they provide the developer with a description of all the significant requirements adequate for the design and implementation of the software.

Most object-oriented analysis methods described in the text books, such as [13], concentrate on modeling facilities, especially conceptual modeling (e.g. object modeling). In addition to that, the methods have some notations for representing dynamic behavior and data transformations in the system. However, the requirements representation is only one part of the requirements definition. In particular, most of the suggested object-oriented analysis methods provide limited facilities for:

- identifying the requirements based on business area analysis,

- eliciting information related to requirements from people (including fact-finding and validation of results),

- deriving software requirements from system requirements,

- analyzing end-user tasks for user interface specification,

- identifying software development constraints,

- classifying requirements as mandatory or desirable requirements, and

- evaluating the feasibility of requirements.

Standards such as IEEE Standard 830, IEEE Guide to Software Requirements Specification [8] give valuable guide-lines on how to document the requirements. The popular object-oriented methods described in textbooks do not seem to address the documentation of requirements in general and non-functional requirements in particular. These kinds of elements from the traditional software engineering discipline need to be added to object-oriented methods in order to make them applicable to industrial strength system development, which fulfills the requirements necessary for the development of products that match customer needs. 


\section{Process Viewpoint}

Successful implementation of large software systems requires a well-defined and managed software process. The process descriptions of the textbooks for objectoriented approaches are usually too loosely defined. Some examples of daily problems not addressed by object-oriented methods - in addition to the previously mentioned deficiencies in the requirements definition process - are:

- Detailed document templates with check-lists for the artifacts are not provided.

- Specification practices of the user interface are often neglected, although usability is one of the most important elements of the system from the user's point of view.

- Software architecture guidelines are vague, and rules for decomposing a large system into processes and libraries are not presented.

- Project management issues are not discussed in detail. However, OOSE and Fusion provide some tips for project planning.

- Although most of the effort is put in modifying the existing software, all existing methods provide proper support for the development from new only. Jacobson et al. [10] discuss this problem extensively, but provide no 'silver bullet' for solving it.

A process model suitable for developing large systems must scale up so that the complexity of the system and the implementation projects can be managed. The process must be defined well enough to be repeatable and produce reusable artifacts that can be processed in future projects. Flexibility in division of labor is also important, especially in large projects, where specialization is required to successfully carry out various activities such as analysis, architectural design, and user interface specification. As an example, Jacobson et al. explain the difference between the OOSE book and their Objectory process description: The book provides only fundamental ideas of the process and a simplified version of it; a detailed process description with examples takes up about 1200 pages [10]. Our experiences of adapting OMT to OMT++ support this view; a lot of effort is needed to establish a solid software process documentation that is detailed enough.

A detailed process description does not guarantee success, though. The greatest challenge also in object-oriented software development is the institutionalization of the new process model, which means major changes in work methods. Successful institutionalization requires strong and large management commitment, adequate funding, readiness for change, and resources for training and support. However, these outward circumstances are not available for all software engineering organizations.

Many organizations claim that they use some methodology such as Structured Analysis/Design, but very few of them really follow the process model. The 'use' of the methodologies usually means that some part of the modeling facilities (such as object diagrams) are used. Bill Curtis has estimated that about $75 \%$ of organizations 
were at initial level 1 of the Capability Maturity Model for Software, CMM, in 1994 [7]. This leads to the conclusion that about $75 \%$ or organizations starting to use object-oriented approaches will face severe problems when taking them into use. Software process models based on object-orientation (or any models) cannot be institutionalized if there are no feasible ways of introducing changes into the organization and verifying their implementation.

In order to facilitate institutionalization, the object-oriented methods need to be adapted to fit into the organization's existing processes. Obviously, they also need to be extended to support the individual needs of the company's software development, such as the large system issues. In the development of OMT++, we have recognized that software engineers need detailed guidelines for implementing features effectively in a large existing system. There are close connections between the four important elements in software development: software process, software architecture, software components, and the tools used, as depicted in Figure 3. A process model explains the chain of activities leading to achievement of the expected artifacts. A model of the system architecture is needed to illustrate the implementation structure of the system: What should be modified, and how new functionality should be added. The architecture of the system should be supported by a suitable set of components forming a platform for applications, and tools should be available for making everything as automated as possible.

The existing object-oriented approaches are on a general level and they do not usually rely on any particular software architecture model. We have successfully integrated the process model in an architecture framework based on the Model-View-Controller approach in OMT++ [9]. In addition, we have integrated tools such as a GUT builder together with the process model [12]. Our experiences suggest that process models, architecture models, components, and tools should not be considered as separated issues; they should be integrated in an integral whole. An approach called design patterns [6] seems a promising way of documenting the experience related to integrating these elements of software development.

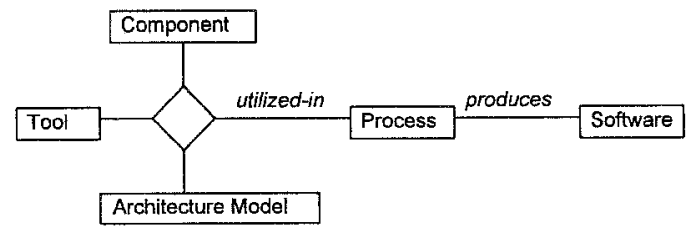

Fig. 3. Elements of software development. 


\section{$4 \quad$ Technology Viewpoint}

The right technology solutions are essential for the success of the product in the long run. This is particularly true for large systems which may live for decades. Because of the rapid development of software technology, any part of the current systems may need to be totally rewritten within the next ten years. We may not be able to avoid that, but we may be able to avoid another risk: choosing immature technology that the customers do not want to buy now or even in a couple of years. If we loose a customer once due to unsuitable technology, our window of opportunity may close, pushing us out of the market place.

From the software supplier's point of view, the main benefits of object technology are derived from the expected improvements in the productivity of work. The elements of object-technology used in carrying out that work consist of:

- Computer-Aided Software Engineering (CASE) tools, and

- components (both Commercial-off-the-shelf (COTS) and internal reusable components).

Although tools are getting better all the time, they cannot be considered mature in general. CASE tool vendors have problems in implementing support for methods due to the absence of rigorous semantics. For instance, code generation and reverse engineering facilities of object-oriented design CASE tools are moderate. Another problem is capacity: Large-scale object models are not truly supported, because the methods do not support them either.

Good programming environments for $\mathrm{C}++$ with all necessary facilities, such as compilers, symbolic debuggers, and browsers, are available. Yet the standardization situation of the $\mathrm{C}++$ programming language is a problem. Features such as templates and exceptions are not properly standardized, and the implementations of the compilers have deficiencies. These deficiencies decrease the portability of systems implemented in $\mathrm{C}++$. In order to minimize the problems related to the immature definition of $\mathrm{C}++$, the use of these $\mathrm{C}++$ features should still be avoided.

Today, implementation tools can probably be selected so that the selected tools are still supported after, say, five years. The main stream object-oriented programming languages, $\mathrm{C}++$ and Smalltalk, seem to be safe selections due to extensive support of tool vendors. Selecting any other object-oriented programming language to develop systems - which are maintained for years - is a risk.

The number of available COTS components is strongly dependent on the programming language used. $\mathrm{C}++$ is often the best choice, since many of the component interfaces are defined using $\mathrm{C}$ programming language or $\mathrm{C}++$. An important factor in large scale development is that the internal components can be 
used now and in the future. To make this possible, only tools and programming languages having high credibility should be used.

Most customers are not interested in the implementation tools used in the development of their system. However, the customers want to know that the credibility of the used technology is adequate. From the customer's point of view, the most important third-party software is the database management system. Object databases are considered to replace relational databases like object-orientation is replacing functional decomposition. Although object databases have several advantages, they are not considered better than relational databases in all types of applications [1]. An important commercial aspect is that many customers of large systems already have some legacy system in place. It is likely that they are not even willing to administer object databases in the near future. It will take some time before the credibility of object database products is so high that customer organizations are willing to include them in their information management policies. This is an example of a solution that is attractive for the development organization but may not be so attractive for practical reasons.

\section{$5 \quad$ Personnel Viewpoint}

Competent and motivated personnel is, no doubt, one of the most important success factors of any expert organization. There are great differences in the individual productivity of work depending on the experience and skills of software engineers. Attitudes and the culture created by personnel contribute strongly to the future of the organization: Readiness for change and for learning new things is important in software engineering, where new technology brings changes continuously. On the other hand, positive changes in processes often increase motivation, if the pace of the changes is not too fast.

Object-orientation also represents a change for many companies and individuals. Currently, it seems to be quite difficult to recruit personnel with a suitable skill-base for object-oriented software development. It may be relatively easy to find a $\mathrm{C}++$ programmer, but although the programmer knows the syntax, he or she may not have internalized the idea of object-oriented programming in the first place. Maybe the 'worst case' is an experienced C++ programmer who has not internalized the concepts of object-oriented development, but who has a lot of experience of the 'wrong' way of designing $\mathrm{C}++$ programs. In any case, an employer cannot be really sure about the object-oriented capabilities of job seekers. We have found it necessary to give all newcomers extensive training in object-oriented thinking, analysis, design, and programming.

It is unfortunate that there is no one or even a few winning 'industrial methods' among object-oriented approaches. OMT seems to be popular when considering the notations used in software engineering articles, for instance. However, none of the methods is as dominating as Structured Analysis/Structured Design (SA/SD) among approaches 
based on functional decomposition. Furthermore, when we consider the fact that the methods are not detailed enough to establish a repeatable process, it is obvious that any employer faces problems when trying to recruit a software analyst or designer who could immediately start using objects in a productive way. This jeopardizes the first schedule-critical projects implemented by newcomers.

An organization developing large systems needs to assure that the capabilities of the personnel are adequate. Since object technology is still a new thing for many companies and university training programs, it is harder to find suitable people for projects implemented using object technology than for projects using traditional methods.

\section{Software Assets Viewpoint}

In OMT++, a feature is a product characteristic offered to customers which fulfills a set of customer requirements. A feature may consist of other features, i.e. the concept is hierarchical. Features often correspond to a set of use cases, but features are not only functional. A feature may be, for instance, a different solution to the same functional need, such as an open communications interface instead of an existing proprietary interface.

Large systems, such as the Nokia OMC, are developed in increments so that new features are designed on top of the existing system. Features are the units that are analyzed, designed, implemented, and tested. The mass of code and related documentation such as specifications, design documents, test cases, and customer documents, comprise the software assets of the company that are needed to develop new features. The software development of large systems relies extensively on the large-scale reuse of software assets. If they deteriorate, their reuse will become less productive, and the quality of the software will decline. The future of the organization will thus be endangered if the software assets are not properly managed.

Large systems need to be decomposed into sub-systems in order to manage their complexity. The need for a proper product breakdown structure is two-fold. First, we need to manage the set of features provided by a complex system through a structure mapped to the customers' needs. Only if the features of the product are understood as a whole, the evolution of the product parts can be managed consistently. If a holistic view to the product is missing, it is quite impossible to establish any clear strategy that would ensure that the system is competitive also in years to come. Secondly, we need to decompose the technical complexity into manageable pieces in order to make the construction of the system possible.

The object-oriented methods today give little support for managing the characteristics of a large system. Decomposition into sub-systems is typically seen only in the design phase, although the classification and management of requirements call for a product breakdown structure already in the analysis phase. Some methods, such as Coad- 
Yourdon OOA [5] and OOSE [10], provide facilities for decomposing the object model into parts (called subjects by Coad and Yourdon and sub-systems by Jacobson et al.) but even they do not seem to give any practical guidelines on how to allocate functional requirements to sub-systems. As a consequence, the traceability of functional requirements is lost because they cannot be traced to sub-systems.

It is not feasible to decompose large systems into sub-systems in the design phase so that one object class contains all behavior (i.e. attributes and operations) related to it. One 'real world' object may be viewed from dozens of perspectives and it may have hundreds of operations and attributes in a large system. The same operation names may even have different meanings depending on viewpoints. On the other hand, independence of decomposed parts is one of the golden rules of any architecture design. In large system development, dozens of software development groups might need to access the same entity object class if all behavior relating to it were centralized into one single class allocated to one sub-system. That would make the maintenance of these software assets quite impossible. The same goes for the division of labor in software development.

In OMT++, the system is decomposed into sub-systems, which are called service blocks, first in the analysis phase. The sub-system division is mainly based on the views that the users of the system have of objects of the problem domain. This product breakdown structure makes it possible to develop service blocks independently and coordinate their dependent product characteristics. In the design phase, service blocks providing implementation services are created in order to establish a layered architecture. They provide services for application designers. Each service block in the design phase consists of program blocks, which are either libraries or processes. The program blocks are the key-elements in software configuration management, and they consist of files to be managed by revision control mechanisms. This product breakdown structure is depicted in Figure 4.

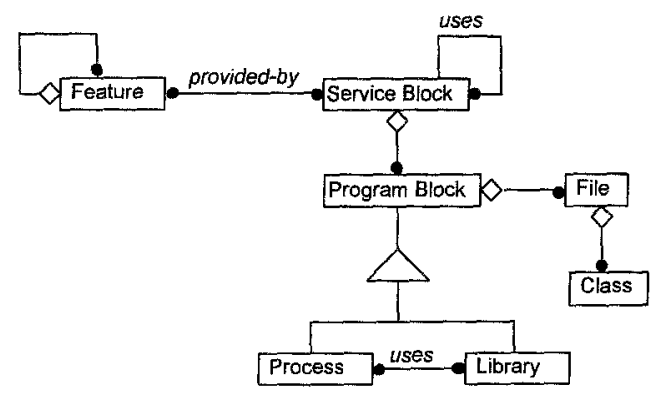

Fig. 4. Concepts of product breakdown structure in OMT++.

Large system development with OMT++ is thus based on features which are allocated to service blocks, which in turn are implemented by communicating processes developed using a set of libraries. Unfortunately, such key-concepts are almost totally 
missing from existing methods, or they are supported only by 'work-arounds'. For instance, there is no notational support for all these concepts. It goes without saying that traceability issues or pragmatic guidelines are not addressed by methods such as OMT, OOSE and Fusion. As emphasized earlier in this paper, the methods described in the respective text books concentrate on developing software from new; maintenance of software assets is not properly described in them.

However, object-oriented approaches to software development offer a good foundation for creating software based on stable concepts expressed in terms of objects. The main challenge faced in large-scale use of these methods is scaling them up. In addition to that, analysis and design models need to be separated in order to allow easy and secure updating of both of them as enhancements and new features are implemented. All document templates need to be considered from the maintenance point of view: How easy it is to add new functionality, which other models need to be updated, etc. These are the challenges of methodologists who deal with large object projects aiming at enhancing the reuse of software assets.

\section{$7 \quad$ Conclusions}

Nokia Telecommunications and Nokia Research Center have developed a methodology called OMT++ [2], which supports the development of the large systems in particular. OMT++ is based on OMT, OOSE and Fusion. The observations of this paper are based on experiences of developing OMT++, and on the information provided by text books about these popular methods [13], [10], [4]. The findings concentrate on situations where the methods are applied to large projects. However, many of the findings apply to small organizations as well.

Object technology has several advantages, but many unresolved issues need to be addressed before it can be fully utilized in the development of large-scale systems. In this paper, these issues were discussed from perspective of the success factors that are important for an organization developing large applications. The main findings were:

- From the product perspective, object-oriented analysis methods need to be supplemented with practices for identifying and documenting non-functional requirements as well. Business process modeling seems to be a suitable front end for the requirements definition process; it helps in finding the functional requirements in particular. The modeling facilities of object-oriented methods seem to be well suited for this kind of business modeling.

- From the process perspective, the object-oriented methods are too loosely defined if a repeatable, managed process is what is expected. The object-oriented methods support development from new, but give little support for incremental development of large systems. Object-orientation calls for a new way of thinking, and this, in turn, requires that the organization can manage the change. The immaturity of most software engineering organizations causes trouble in the 
institutionalization of object-oriented technology. Its buy-in can be facilitated by pragmatic guide-lines which not only define the process steps, but also integrate the use of tools, software components, and the architecture model in the process.

- There are two technology perspectives: that of the customers and that of the development organization. The tools used in development are maturing, but several problems still exist, mostly related to the loose definition of methods. Those technology solutions that are attractive to development organizations, such as object databases, may not be so attractive for user organizations. Correct timing and the selection of a product with high credibility are of vital importance to long term success of systems which may be used and developed for decades.

- The new way of thinking is a challenge from the personnel perspective - both for employees and employers. Individuals need to develop their skill-base in order to be productive in utilizing the possibilities provided by objects. Employers will face problems in recruiting for some years, in other words, as long as the majority of job-seekers has inadequate knowledge of object-orientation. Large projects requiring a lot of resources need to establish wide training programs in order to create a suitable skill-base for the organization.

- Object-oriented methods are problematic from the software assets perspective as far as the development of large systems is concerned. Basic concepts of large systems such as feature, process, and library do not constitute a part of the concepts of the methods. In practice, support for requirements traceability ends when a system is divided into sub-systems. Since concepts, such as process, are missing, no pragmatic support is provided for mapping objects to processes by methods such as OOSE, OMT or Fusion. They are mostly meant for developing one application from scratch. A solid product breakdown structure needs to be established in order to manage the functionality provided by the product as a whole also in the long run. This kind of manageable structure enables productivity gains through the large-scale reuse, and provides a basis for the product structure which can be enhanced by new features for years to come. When the functionality becomes manageable, another great challenge is posed by the system architecture. The existing methods do not give clear guide-lines for developing a robust software architecture for systems having hundreds of communicating processes and thousands of classes.

It seems quite obvious that object-orientation will be the mainstream software development approach of this decade. Many challenges still call for pragmatic solutions in order to make objects into productive building blocks for large systems.

\section{Acknowledgments}

This paper has been reviewed by my colleagues Ari Aalto, Ari Jaaksi, Hannu Pahkala and Sari Hänninen from Nokia Telecommunications, by Ilkka Haikala from Tampere 
University of Technology, and by Kalle Lyytinen from the University of Jyväskylä. I would like to thank them all for their valuable comments on the earlier versions of this paper. I would also like to thank the reviewers, Pasi Rajala and Pertti Tapola from Nokia Telecommunications for the valuable debates which have helped me in preparing this paper.

\section{References}

1. A. Aalto: Data Management Solutions in an Object-oriented Application. Licentiate thesis, Tampere University of Technology, 1994 (in Finnish).

2. J-M. Aalto: A. Jaaksi: Object-oriented Development of Interactive Systems with OMT++. In: R. Ege, M. Singh, B. Meyer (Eds.): Proceedings of the Fourteenth International Conference TOOLS Santa Barbara 1994. Prentice-Hall, 1994.

3. J. Brackett: Software Requirements. SEI Curriculum Module SEI-CM-19-1.2, Carnegie-Mellon University, 1990.

4. D. Coleman, P. Arnold, S. Bodoff, C. Dollin, H. Gilchrist, F. Hayes, P.Jeremaes: The Fusion Object-Oriented Analysis and Design Method. Prentice Hall, 1994.

5. P. Coad, E. Yourdon: Object-oriented Analysis. Prentice-Hall, 1991.

6. E. Gamma, R. Helm, R. Johnson, J. Vlissides: Design Patterns: Elements of Reusable Object-oriented Software. Addison-Wesley, 1994.

7. W. Gibbs: Software's Chronic Crisis. Scientific American. September, 1994.

8. IEEE: IEEE Guide to Software Requirements Specification. ANSI/IEEE Std 8301984. IEEE, 1984.

9. A. Jaaksi: Implementing Interactive Applications in C++. Software Practice and Experience. John Wiley \& Sons, 1995 (in print).

10. I. Jacobson, M. Christerson, P. Jonsson, G. Övergaard: Object-oriented Software Engineering - a Use Case Driven Approach. Addison-Wesley, 1992

11. I. Jacobson, M. Ericsson, A. Jacobson. The Object Advantage. Business Process Re-engineering with Object Technology. Addison-Wesley, 1994

12. V. Punkka: Intelligent Reusable User Interface Components. The X Resource, Issue 13. O'Reilly \& Assoc. Inc., 1995.

13. J. Rumbaugh, M. Blaha, W. Premerlani, F. Eddy, W. Lorensen: Object-oriented Modeling and Design. Prentice-Hall, 1991. 